\title{
Expression of the Sonic Hedgehog pathway components in clear cell renal cell carcinoma
}

\author{
ANNA KOTULAK-CHRZASZCZ ${ }^{1}$, JAKUB KLACZ ${ }^{2}$, MARCIN MATUSZEWSKI ${ }^{2}$, \\ ZBIGNIEW KMIEC $^{1}$ and PIOTR M. WIERZBICKI ${ }^{1}$
}

\author{
${ }^{1}$ Department of Histology, Faculty of Medicine, Medical University of Gdansk, Gdansk 80211; \\ ${ }^{2}$ Department of Urology, Faculty of Medicine, Medical University of Gdansk, Gdansk 80402, Poland
}

Received December 13, 2018; Accepted August 6, 2019

DOI: 10.3892/ol.2019.10919

\begin{abstract}
Clear cell renal cell carcinoma (ccRCC) is the most common and the most aggressive histopathological subtype of kidney cancer, with patients exhibiting high mortality rates for metastatic tumors. The Sonic Hedgehog (SHH) pathway serves a crucial role in embryonic development. The abnormal activity of SHH signaling is observed in a broad range of malignancies. However, its role in ccRCC is still undetermined. The aim of the present study was to assess the expression of the SHH pathway genes in ccRCC. Neoplastic and morphologically unchanged kidney tissues were obtained during radical nephrectomy from 37 patients with ccRCC. The SHH, PTCH1, SMO and GLII mRNA levels were assessed using the reverse transcription-quantitative PCR. Western blot analysis was used to assess the full-length and C-terminal SHH protein level. The mRNA levels of $S H H, S M O$ and $G L I 1$ were approximately 2-, 2,5and 7-fold higher in ccRCC tissue compared with control kidney tissue, respectively. Correlational analysis between the mRNA levels of SHH pathway genes and patients' clinicopathological factors revealed decreased and increased mRNA levels of PTCHI and SMO respectively, in tumor samples derived from older patients (age >62). Furthermore, the level of C-terminal SHH protein in ccRCC samples was significantly lower in a group of males compared with females. No correlation was exhibited between molecular data and patient survival. Western blot analysis indicated a 3-fold higher level of SHH full-length protein, and a 4-fold lower level of the C-terminal SHH protein domain, in ccRCC
\end{abstract}

Correspondence to: Mrs. Anna Kotulak-Chrzaszcz, Department of Histology, Faculty of Medicine, Medical University of Gdansk, 1 Debinki Street, Gdansk 80-211, Poland

E-mail: annkot@gumed.edu.pl

Key words: clear cell renal cell carcinoma, gene expression, quantitative PCR, western blot analysis, Sonic Hedgehog pathway, Sonic Hedgehog Signaling Molecule, Patched 1, Smoothened, Frizzled Class Receptor, GLI Family Zinc Finger 1, prognostic factors tumor tissues compared with normal kidney samples. The current study indicated an involvement of the SHH pathway in ccRCC development.

\section{Introduction}

Kidney cancers are among the 10 most frequently diagnosed malignancies worldwide (1). Clear cell renal cell carcinoma (ccRCC) represents the most common as well as the most aggressive histopathological subtype (2). The 5-year survival rate for systematically spread kidney cancers is approximately $12 \%$ (3). Therefore, there is an urgent need for research which may establish new molecular targets responsible for ccRCC initiation and progression (4).

The Sonic Hedgehog (SHH) pathway plays an important role during embryogenesis and in the maintenance of tissue homeostasis during postnatal life (5-7). SHH full-length protein (424aa) is cleaved intracellularly (8) to provide two biologically active products. $\mathrm{C}$-terminal $\mathrm{SHH}$ protein (227aa) acts as an autoprocessing domain, while N-terminal $\mathrm{SHH}$ protein (174aa) is secreted and may act as a ligand either via auto- or paracrine signaling (9). The binding of $\mathrm{N}$-terminal SHH molecule to the Patched-1 (PTCH1) cell membrane receptor initiates intracellular signal transduction through Smoothened (SMO) co-receptor and GLI zinc finger proteins, which, acting as transcription factors, activate transcription of several target genes, e.g. $M Y C N, b c l 2$ or VEGF (10).

Aberrant expression of SHH, PTCHI, SMO and GLII genes associated with cancer progression and patients survival has been reported in a broad range of human malignancies such as basal cell carcinoma (11), breast cancer (12) and other neoplasms $(13,14)$. However, the results of SHH pathway genes expression, both at the mRNA as well as protein level, in ccRCC human tissues are contradictory (15-17).

Therefore, we decided to perform the analysis of the expression of SHH pathway genes at the mRNA level in ccRCC tumor and paired unchanged kidney tissue. Moreover, we assessed the level of full-length SHH protein as well as the C-terminal SHH domain protein in kidney tumor lysates by western blot method. The results were statistically analyzed in terms of clinicopathological features of ccRCC patients and their overall survival (OS). 


\section{Materials and methods}

Patients and samples. The ccRCC tumor tissue and morphologically unchanged kidney samples were obtained during radical nephrectomy from 37 patients operated in the Department of Urology, Medical University of Gdańsk, Poland. The exclusion criteria for the study were: diagnosis of VHL disease, multifocal or/and bilateral kidney tumors, other than ccRCC histological subtypes of RCC. The study was approved by the local Ethics Committee (decision no. NKEBN/4/2011 and NKBBN/370/2016); written consent was acquired before the surgery from each patient. The clinicopathological features of the patients were presented in Table I.

Material acquisition. Small (ca. 7x2, 7x2, 7x2 mm) pieces of ccRCC and morphologically unchanged tissues (resected from at least $2 \mathrm{~cm}$ from the tumor) (18) were placed into test tubes in the operating theater, no longer than $20 \mathrm{~min}$ after kidney resection. One of the three sectioned pieces of obtaining material was placed into about 5 volumes of RNA later (Ambion Inc.), and after $24 \mathrm{~h}$ placed at $-80^{\circ} \mathrm{C}$ until analyzed by qPCR and western blotting. The other two samples of tumor tissue were fixed in $4 \%$ buffered formalin solution, embedded in paraffin, sectioned and stained with hematoxylin and eosin (H\&E) for histopathological assessment. The tumor samples were subjected to qPCR and WB analyses only if $>60 \%$ cells in the respective histological sections in tumor samples presented characteristic features of ccRCC while all cells of unchanged (control) samples presented normal morphology $(18,19)$. If both conditions were not fulfilled, the patient was excluded from the study.

Total RNA isolation. Total RNA from the collected samples was isolated using the ExtractMe Total RNA kit (Blirt) according to the manufacturer's protocol. The collected samples were homogenized in $2 \mathrm{ml}$ tubes with $300 \mu \mathrm{l}$ lysis buffer and ceramic beads using the MagnaLyser apparatus (Roche Diagnostics) for $40 \mathrm{sec}$ at $6,000 \mathrm{rpm}$. The obtained RNA was dissolved in $70 \mu 1$ of nuclease-free water. The quantity and quality of RNA were measured with a spectrophotometer (NanoDrop ND 1000; Thermo Fisher Scientific). RNA samples were stored at $-80^{\circ} \mathrm{C}$ until further analysis.

First-strand cDNA synthesis. $1 \mu \mathrm{g}$ RNA was reversibly transcribed using $1 \mu \mathrm{l}$ RevertAid reverse transcriptase (Fermentas; Thermo Fischer Scientific) and 0,5 $\mu \mathrm{g}$ dT18 primers (Sigma-Aldrich, Munich, Germany) in a total volume of $20 \mu \mathrm{l}$. The reaction was performed according to the manufacturer's (Fermentas; Thermo Fischer Scientific) protocol. cDNA samples were collected at $-20^{\circ} \mathrm{C}$ until further analysis.

Assessment of gene mRNA level. The mRNA assessment was performed by the qPCR technique. Primers' sequences were designed using the Primer-BLAST software; their concentrations, as well as experimentally established reaction conditions, are presented in Table II. The measurements were performed in duplicate using $1 \mu 1$ of $4 \mathrm{x}$ diluted cDNA and SensiFast Sybr ${ }^{\mathrm{TM}}$ No-Rox kit (Bioline) chemistry in a total volume of $10 \mu \mathrm{l}$. The reaction was conducted on separate PCR plate (4titude) for each gene with negative control (water instead of cDNA) and 10x diluted pooled cDNA as a precision control. StepOne Plus apparatus with accompanying software ver. 2.3 (Life Technologies; Applied Biosystems) was used for the amplification process and data analysis. Geometric mean of $\mathrm{Ct}$ (threshold cycle) values for each gene was normalized to the reference gene (GUSB), according to our previous normalization study on ccRCC (20), using the Livak's equation (21): $X=2^{\Delta \mathrm{Ct}}$, where $\mathrm{X}$ stands for expression of gene $\mathrm{Y}$ and $\Delta \mathrm{Ct}=\mathrm{Ct}$ Gusb-Ct gene Y. Obtained raw expression data for each tumor sample were calibrated to average expression data of control samples (fold change; control sample $=1$ ).

Western blot analysis. Renal biopsies were gently fragmented with Mammalian Cell Extraction Kit (Biovision, Inc.) in tissue homogenizer MagnaLyzer (Roche Diagnostics). Measurement of protein concentration in homogenates was performed by Bradford protein assay with Coomasie Brillant Blue dye (Sigma-Aldrich). Bovine serum albumin (BSA; Sigma-Aldrich) was used as a standard for the preparation of the calibration curve. The proteins were next separated by their weight using SDS-PAGE (12\%; Mini-Protean Tetra System; Bio-Rad). Electrotransfer from an electrophoretic gel to PVDF membrane was carried out in the Mini-Protean Tetra System apparatus (Bio-Rad). The membrane was next incubated with 3\% BSA (Sigma-Aldrich) in TBS (Tris-buffered saline; $\mathrm{pH}$ 7.5) at room temperature (RT) for $1 \mathrm{~h}$. To detect SHH protein, PVDF membrane was first incubated with the monoclonal rabbit anti-human SHH antibody [EP1190Y] (dilution 1:1,000; Abcam) overnight at $4^{\circ} \mathrm{C}$, and then with the peroxidase conjugate polyclonal anti-rabbit IgG produced in goat [A6154] (dilution 1:10,000; Sigma-Aldrich) for $2 \mathrm{~h}$ at RT. After each incubation step, the TBST solution ( $0.1 \%$ Tween-20 in TBS) was used for washing the membrane. To obtain the electrophoretic bands Chemiluminescent Peroxidase Substrate (Sigma-Aldrich) was used. Afterward, the PVDF membrane was also incubated with a monoclonal anti-GAPDH peroxidase antibody produced in mouse (dilution 1:50,000; Sigma-Aldrich) for $1 \mathrm{~h}$ at RT to obtain the signal from the reference protein. Densitometric analysis of electrophoretic bands was conducted through the Quantity One Software (Bio-Rad). The values of band intensity $/ \mathrm{mm}^{2}$ for full-length or C-terminal SHH protein were normalized to those from the GAPDH protein examination. Final semi-quantitative results for tumor samples were obtained as a ratio=mean units Tumor $_{\text {I }} /$ mean units Control $_{\text {for full-length or }}$ C-terminal SHH protein.

Statistical analysis. Statistics were performed with the use of GraphPad Prism ver. 5.00 (GraphPad Software, Inc.) and Statistica ver. 13.1 (Statsoft Inc.). To compare clinicopathological and molecular data Wilcoxon signed-rank and Fisher's 2x2 exact tests were used. Any correlation analysis presented in the study was performed by Spearman's test. Kaplan-Meier analysis was performed to verify the associations between obtained molecular data and patients' clinicopathological parameters as well as overall survival. In all statistical analyses, a two-sided $\mathrm{P}<0.05$ was considered as statistically significant with a $95 \%$ confidence interval.

\section{Results}

Clinicopathological characteristics of patients. The study encompassed 37 ccRCC patients, 13 female, and 24 male, 


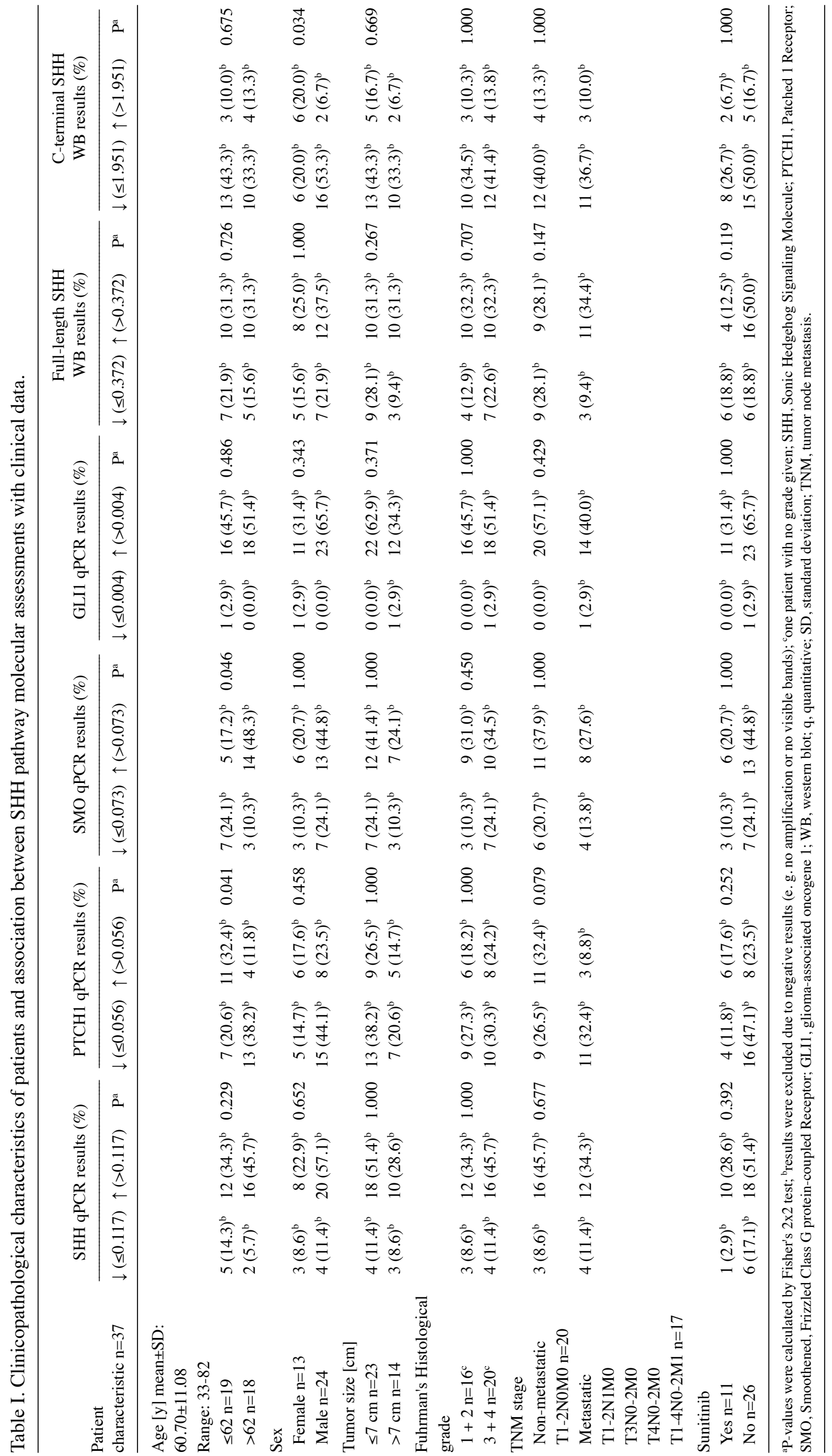




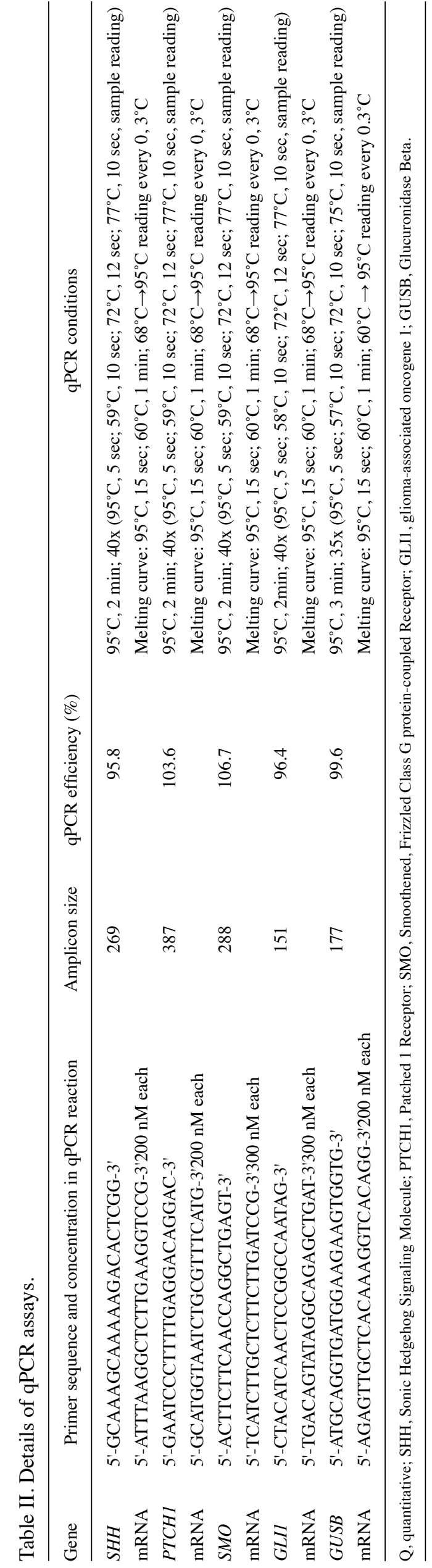

with mean age $60.70 \pm 11.08$ years (Table I). According to AJCC/UICC TNM classification of malignant tumors (1), 17 patients were diagnosed as stage I (T1N0M0), 3 as stage II (T2N0M0), 13 as stage III (T1-2N1M0 or T3N0-2M0) and 4 as stage IV (T4N0-2M0 or T1-4N0-2M1). Histopathological examination of ccRCC tissues indicated 2 patients with grade 1,14 patients with grade 2,12 patients with grade 3 and 8 patients with grade 4 (1 patient with no grade given) following to Fuhrman grading system. For some samples, the results of molecular assessments were excluded due to negative results (e.g. no amplification or no visible bands; Table I). The mean follow-up period was 38 months (range, 3-72). All deaths were associated with ccRCC progression. The median overall survival (OS) rate was 24 months. During follow-up, metastases occurred in 11 patients.

Expression of the SHH pathway genes at the mRNA level. qPCR analysis revealed significantly, approximately 2-,2,5- and 7-fold higher $S H H, S M O$ and GLII mRNA levels, respectively, in ccRCC samples, compared to morphologically unchanged kidney tissue (Fig. 1A, C and D). There were no statistically significant differences between the expression of the $\mathrm{PTCHI}$ gene in cancer and control tissues (Fig. 1B). Correlation analysis between mRNA levels of SHH pathway genes and patients clinicopathological factors revealed lower expression of $\mathrm{PTCH} 1$ as well as higher mRNA level of $S M O$ in tumor samples derived from older patients (age $>62 ; \mathrm{P}<0,05$; Table I). Moreover, the level of C-terminal SHH protein in ccRCC samples was significantly lower in a group of males than females (Table I).

Association between mRNA levels of the analyzed genes. The results of Spearman's test revealed a strong ( $\mathrm{rs}=0.729)$ positive correlation between $S M O$ and GLII expression (Fig. 2F). Moreover, medium positive correlations were observed between the mRNA levels of $S H H$ and $S M O$ (Fig. 2B) as well as $S H H$ and GLII (Fig. 2C) genes ( $\mathrm{rs}=0.561$ and $\mathrm{rs}=0.646$, respectively). A negative correlation was found between PTCHI and SMO (Fig. 2D; rs=-0.579) as well as PTCH1 and GLII (Fig. 2E; rs=-0.378) expression. There was no statistically significant correlation between the expression of $\mathrm{SHH}$ and PTCHI (Fig. 2A) genes.

Semi-quantitative SHH protein level assessment. Western blot analysis demonstrated some differences in full-length and C-terminal SHH protein levels between tumor and control samples. According to Fig. 3, which presents obtained representative electrophoretic bands, in 5/8 matched tissue pairs the level of full-length SHH protein was higher in tumor samples compared to control. A similar or lower level of full-length SHH protein in ccRCC tissues was observed in 3 cases. We also observed a remarkable difference between the C-terminal SHH protein content, which was much higher in control tissues and very low or undetectable in ccRCC samples. These findings were confirmed by the densitometric analysis which revealed approximately 3-fold higher as well as a 4-fold lower level of full-length and C-terminal SHH proteins respectively, in ccRCC tissues compared to control samples $(\mathrm{P}<0.05$; Fig. $4 \mathrm{~A}$ and $\mathrm{B})$. Spearman's test did not show any correlation between $S H H$ mRNA level and neither full-length nor C-terminal SHH protein levels (data not shown). However, there was a positive correlation 

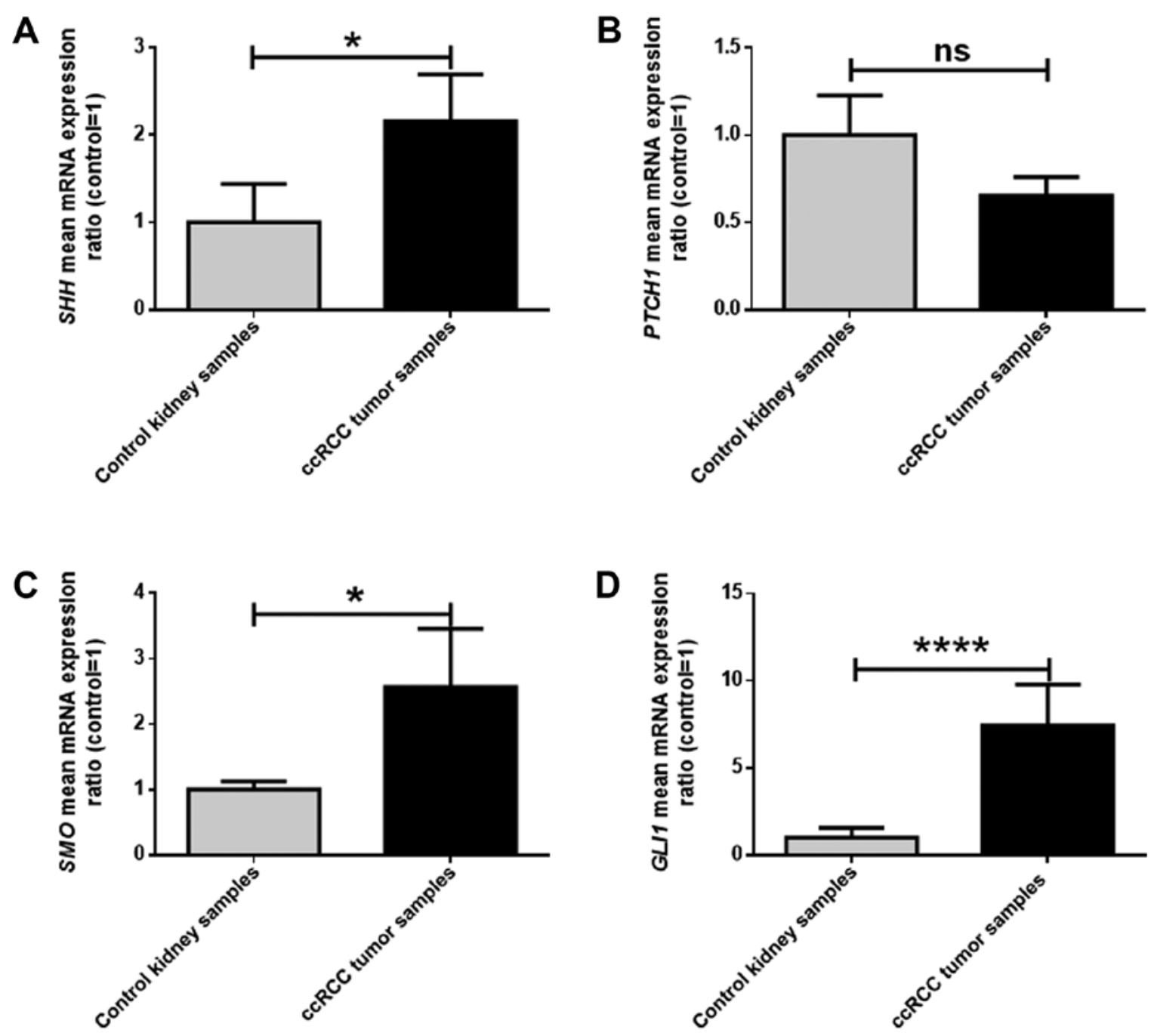

Figure 1. SHH pathway gene mRNA levels in ccRCC tumor samples and morphologically unchanged (control) kidney tissue. (A) SHH, (B) PTCH1, (C) SMO, (D) GLII; mRNA levels in tissue samples were assessed by quantitative PCR. Bars and whiskers represent mean \pm SEM normalized to control kidney samples. ${ }^{*} \mathrm{P}<0.05 ;{ }^{* * * *} \mathrm{P}<0.0001$ between tumor and control samples (Wilcoxon signed-rank test). SHH, Sonic Hedgehog Signaling Molecule; ccRCC, clear cell renal cell carcinoma; PTCH1, Patched 1 Receptor; SMO, Smoothened, Frizzled Class G protein-coupled Receptor; GLI1, glioma-associated oncogene 1.

between the levels of both analyzed SHH protein fragments in cancer tissues, but not in control samples ( $\mathrm{rs}=0,421, \mathrm{P}=0,021$ and, rs $=0,217, \mathrm{P}=0.258$, respectively; Fig. 5).

Survival analysis. The overall survival of patients with ccRCC was strongly associated with higher Fuhrman grading and male sex (Fig. 6A and B). However, TNM staging, $S H H$, PTCHI and SMO mRNA levels as well as full-length and C-terminal SHH protein levels were not correlated with patients' survival (Fig. 6C-H).

\section{Discussion}

It has been suggested that the processes of tumorigenesis and embryogenesis display some similar biological features such as increased cell proliferation, differentiation, and migration (22). Indeed, increased activity of the SHH signaling, which is normally limited to the embryonic development, was also observed in basal cell carcinoma (11), breast (12), colon (13) and gastric (14) cancers. However, the contribution of the SHH pathway to ccRCC development remains unclear.
Thirty-seven ccRCC patients were enrolled in the present study. Although the number of participants is relatively small their clinical-pathological data corresponds with characteristic features of ccRCC reported for larger ccRCC cohorts, e.g. mean age of ccRCC manifestation at 61 (19) with our median age of 62 years old. Most of our patients were males, however the $\mathrm{M} / \mathrm{F}$ ratio (1.83) is comparable $\mathrm{M} / \mathrm{F}$ ratio (1.56) in the USA in 2011 (23).

To the best of our knowledge, the present study seems to be the first to report increased $S H H$ gene expression in ccRCC at the mRNA and protein level. Zhou et al, which evaluated the expression of the main SHH pathway components in 58 cases of ccRCC, indicated the lower level of $S H H$ mRNA in cancer samples compared to normal kidney tissues (16). Possible explanation concerning the discrepancy between Zhou et al and our results may be associated with differences in research methodology, such as different reference gene used in the analysis of the qPCR results. Interestingly, the overexpression of the $S H H$ gene at the mRNA level was observed in non-small cell lung cancer compared to matched normal lung samples derived from 83 patients (24). Moreover, 

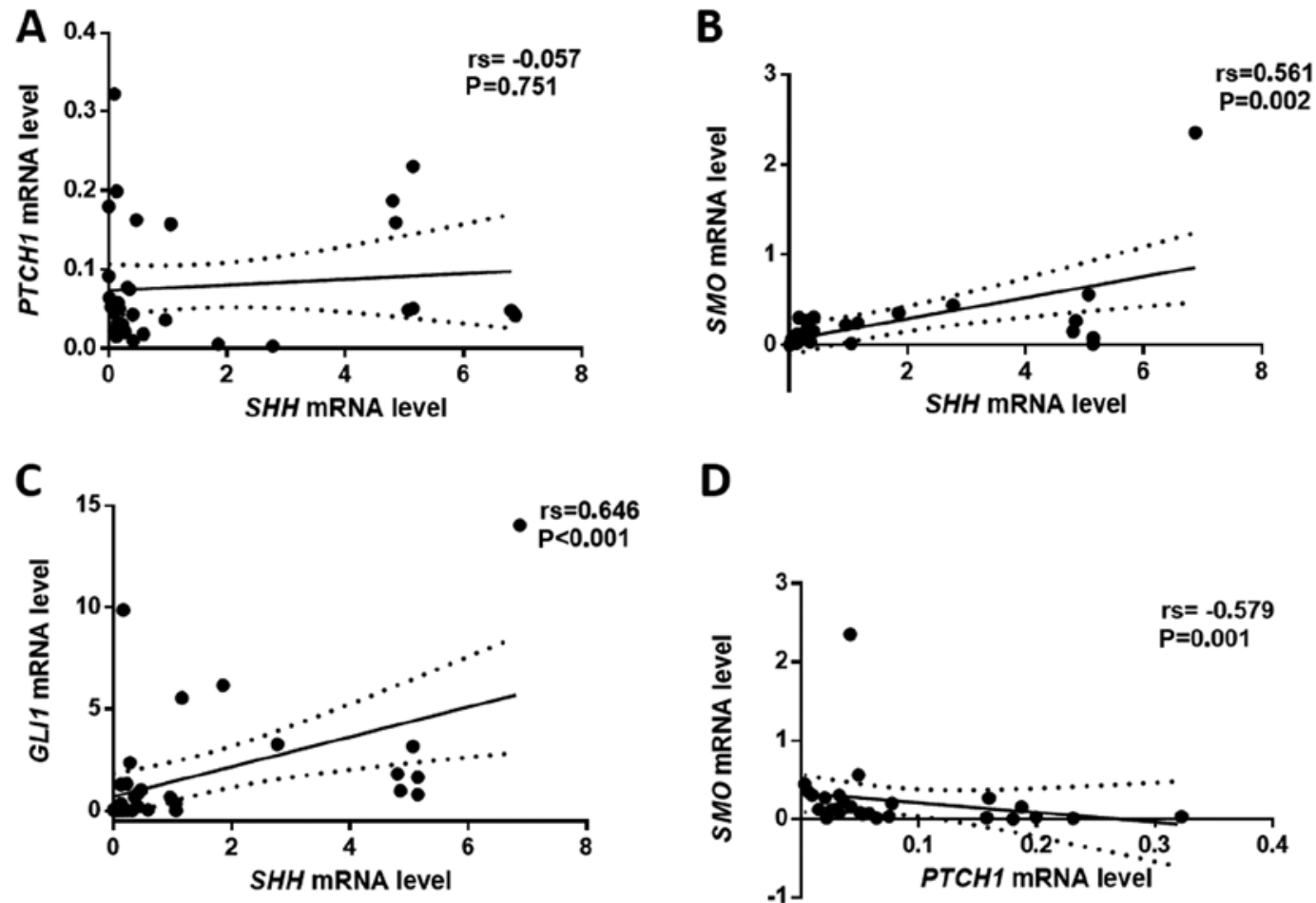

D
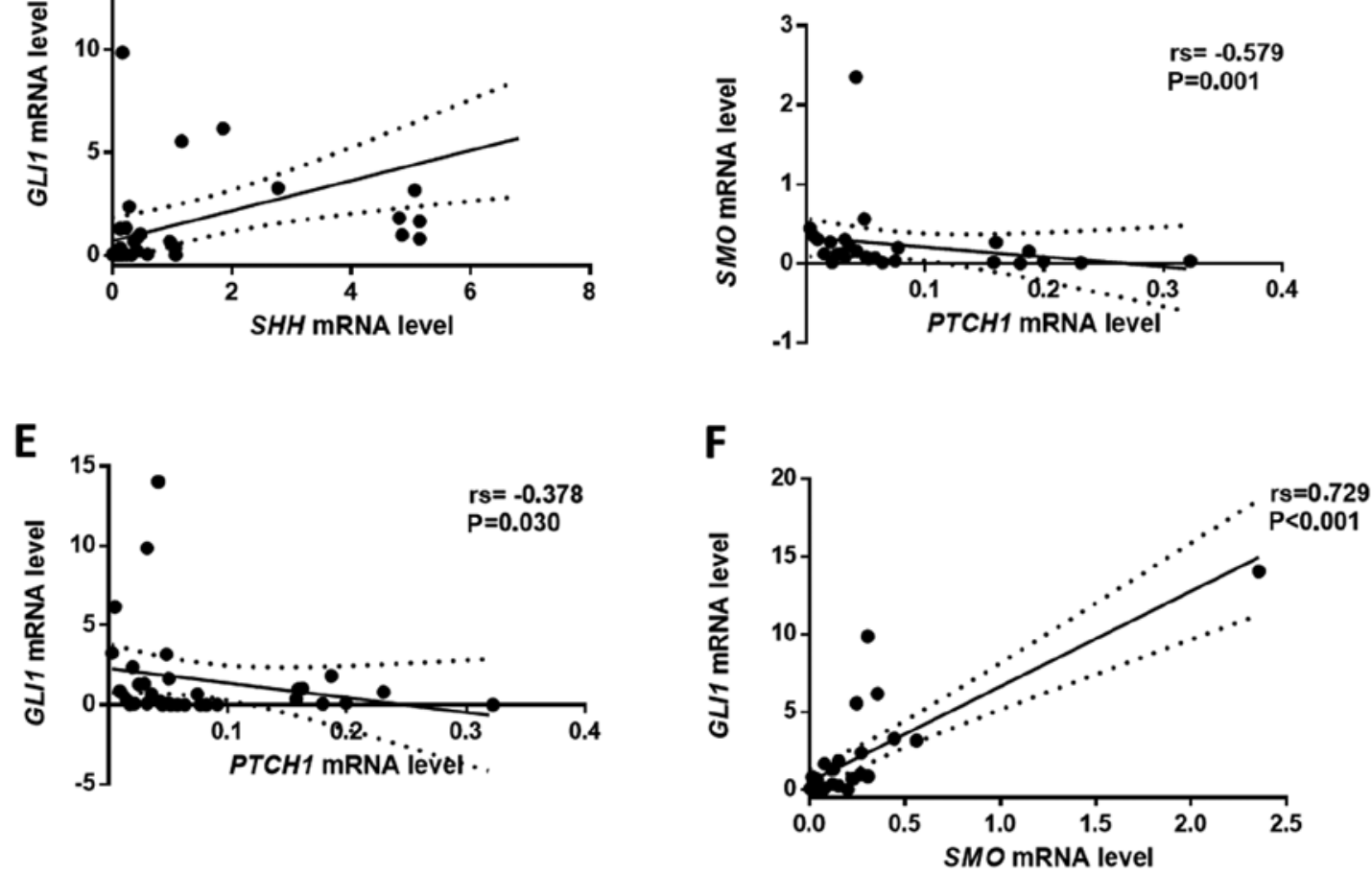

Figure 2. Correlations plots between mRNA levels of the SHH pathway molecules in cancer tissues.P-values were calculated using a Spearman's test. SHH,Sonic Hedgehog Signaling Molecule; PTCH1, Patched 1 Receptor; SMO, Smoothened, Frizzled Class G protein-coupled Receptor; GLI1, glioma-associated oncogene 1.

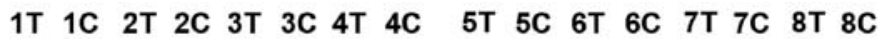

Full-length SHH $51 \mathrm{kDa}$

C-terminal SHH $27 \mathrm{kDa}$

GAPDH $37 \mathrm{kDa}$

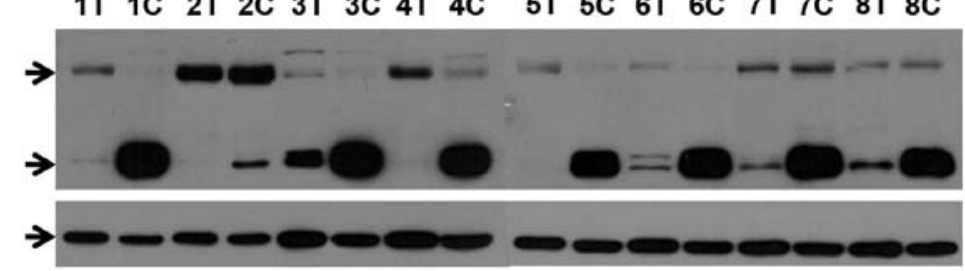

Figure 3. Western blot analysis of full-length and C-terminal SHH protein levels. Representative electrophoretic bands for eight patients (1-8) of full-length (424aa) and C-terminal (227aa) SHH proteins in tumor (T) and control kidney (C) samples. GAPDH was used as a reference protein. In 5 matched tissue pairs, a higher level of full-length SHH protein was indicated in tumor samples compared with the control (patients no. 1,3,4,5 and 6). A similar or lower level of full-length SHH protein in ccRCC tissues was observed in 3 cases (patients no. 2, 7, 8). A marked difference between the C-terminal SHH protein content which was revealed to be increased in control tissues (patients no. 3,6,7 and 8) and significantly decreased or undetectable in ccRCC samples (patients no. 1, 2, 4 and 5). SHH, Sonic Hedgehog Signaling Molecule; ccRCC, clear cell renal cell carcinoma.

a higher level of $S H H$ mRNA was observed in lung tumors assessed as TNM-2 than TNM-1 and cases in which pleural invasion was presented (24). These findings suggest that $\mathrm{SHH}$ mRNA level may act as a potential prognostic factor in lung cancer.
To complete our observation of increased SHH mRNA level in ccRCC, we performed the measurement of full-length and C-terminal SHH protein contents by the western blot method. Our analysis revealed a considerable increase of the full-length SHH protein level, which confirms the results of 

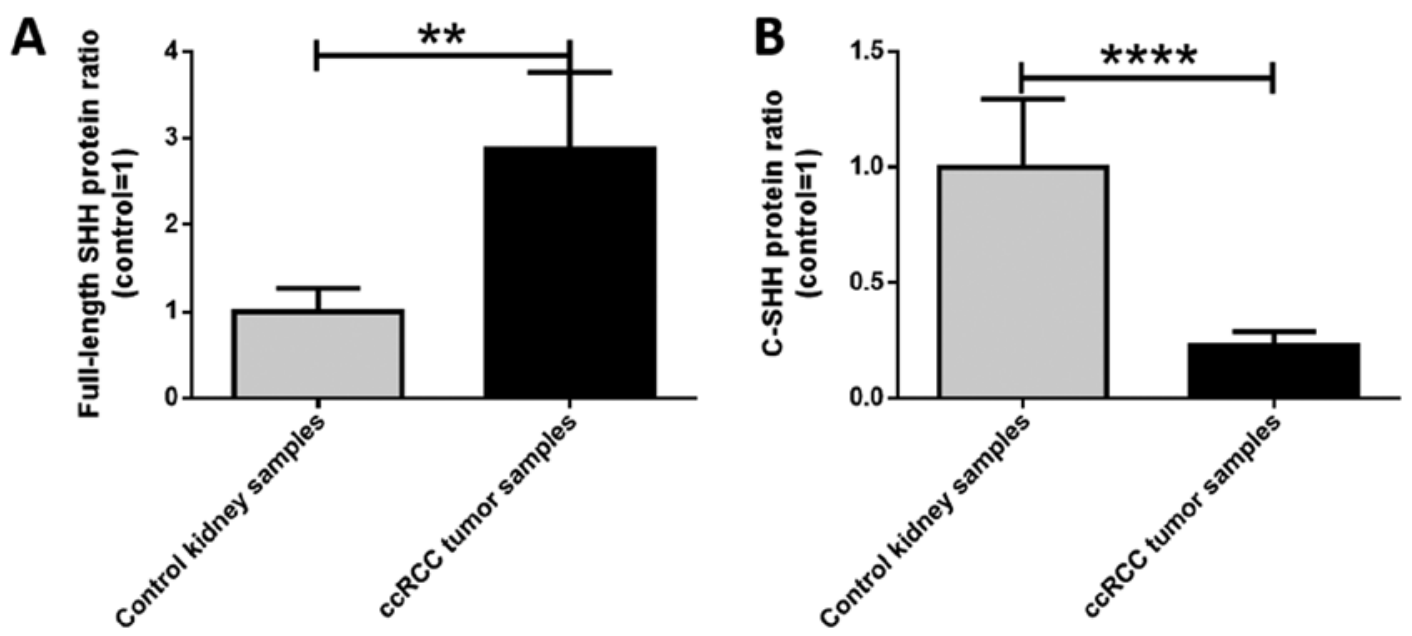

Figure 4. Western blot analysis of (A) full-length SHH and (B) C-terminal SHH protein levels in ccRCC tumor samples and morphologically unchanged (control) kidney tissue. Bars and whiskers represent mean \pm SEM normalized to control kidney samples. ${ }^{* *} \mathrm{P}<0.01 ;{ }^{* * * * *} \mathrm{P}<0.0001$ between tumor and control samples (Wilcoxon signed-rank test). SHH, Sonic Hedgehog Signaling Molecule; ccRCC, clear cell renal cell carcinoma.
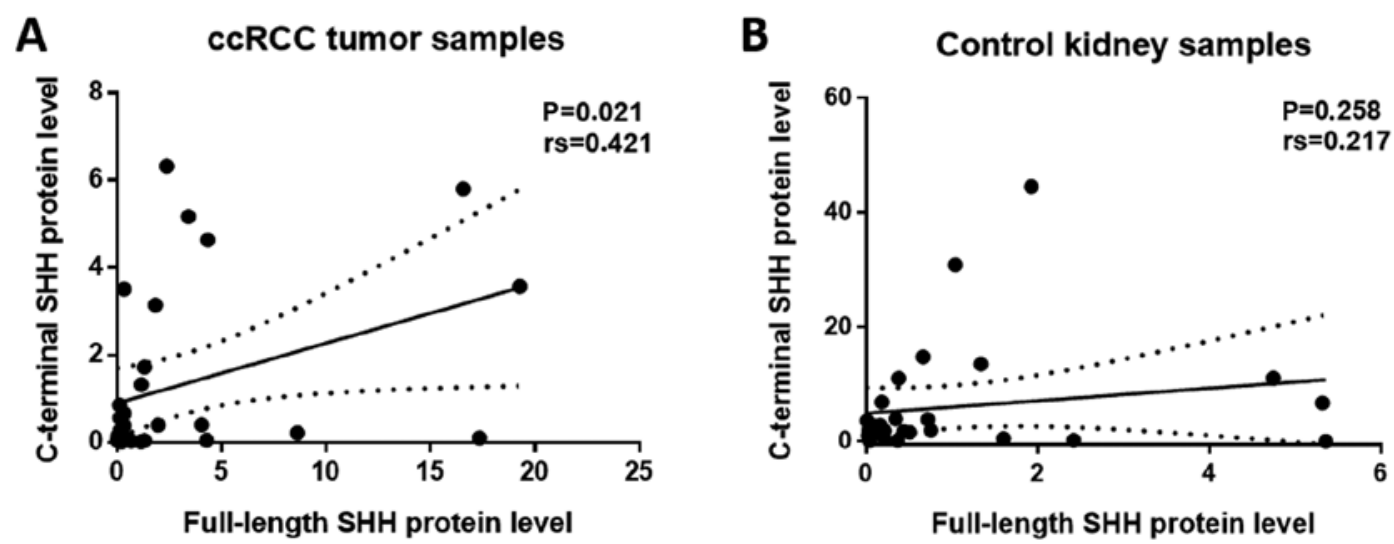

Figure 5. Correlation plots between full-length and C-terminal SHH protein levels in (A) ccRCC tumor samples and (B) morphologically unchanged (control) kidney tissues. P-values were calculated using a Spearman's test. SHH, Sonic Hedgehog Signaling Molecule; ccRCC, clear cell renal cell carcinoma.

the qPCR analysis. We also found a significant decrease of the C-terminal SHH domain in ccRCC tissues, what is the novel observation in cancer tissues. Further experiments are required to find out what is the mechanism of these changes. It has to be mentioned that our analysis did not include the $\mathrm{N}$-terminal SHH domain due to the lack of commercially available highly specific antibodies.

The difference between the level of full-length SHH protein in ccRCC and normal kidney tissue has not been observed so far (9). However, there were some changes at the $\mathrm{SHH}$ protein level in other cancer types. Bian et al (25) examined 142 papillary thyroid carcinoma samples by immunohistochemical (IHC) method. They demonstrated a statistically higher immunoreactivity of full-length $\mathrm{SHH}$ protein in most tumor tissue samples, compared to adjacent non-cancerous thyroid samples as well as the association between $\mathrm{SHH}$ protein level and tumor size, clinical staging, and lymph node metastasis (25). Furthermore, aberrant $\mathrm{SHH}$ gene expression was indicated not only in cancers derived from epithelial cells but also other types of malignancies such as retinoblastoma. IHC staining of 79 retinoblastoma samples revealed that $\mathrm{SHH}$ protein was presented in most cases of neoplastic tissues unlike normal retina samples and high SHH immunoreactivity was correlated with advanced disease status including local invasion and metastasis (26).

The Patched1 (PTCH1) receptor is a 12-pass transmembrane protein, which inhibits Sonic Hedgehog signaling when it is unliganded (27). In ccRCC Zhou et al found a considerable decrease of PTCH1 mRNA level (16). Our study also revealed the tendency towards a lower level of $\mathrm{PTCH} 1$ expression at the mRNA level, but the results are not statistically significant.

The SHH pathway signaling transducer, Smoothened protein (SMO), is the main target for several molecular antitumor agents, which are tested in clinical trials (28). To date, two of them, vismodegib and sonidegib, have been approved by the US Food and Drug Administration (FDA) for treating locally advanced and metastatic basal cell carcinoma (BCC) (29). Our results revealed a statistically significant increase of the $S M O$ gene expression at the mRNA level in ccRCC samples, compared to control tissues. The previous report about the expression of SHH pathway genes in ccRCC tissues did not indicate differences between $S M O$ mRNA level in ccRCC tissues and normal kidney sections (16). However, Dormoy et al observed that cyclopamine, the substance that 

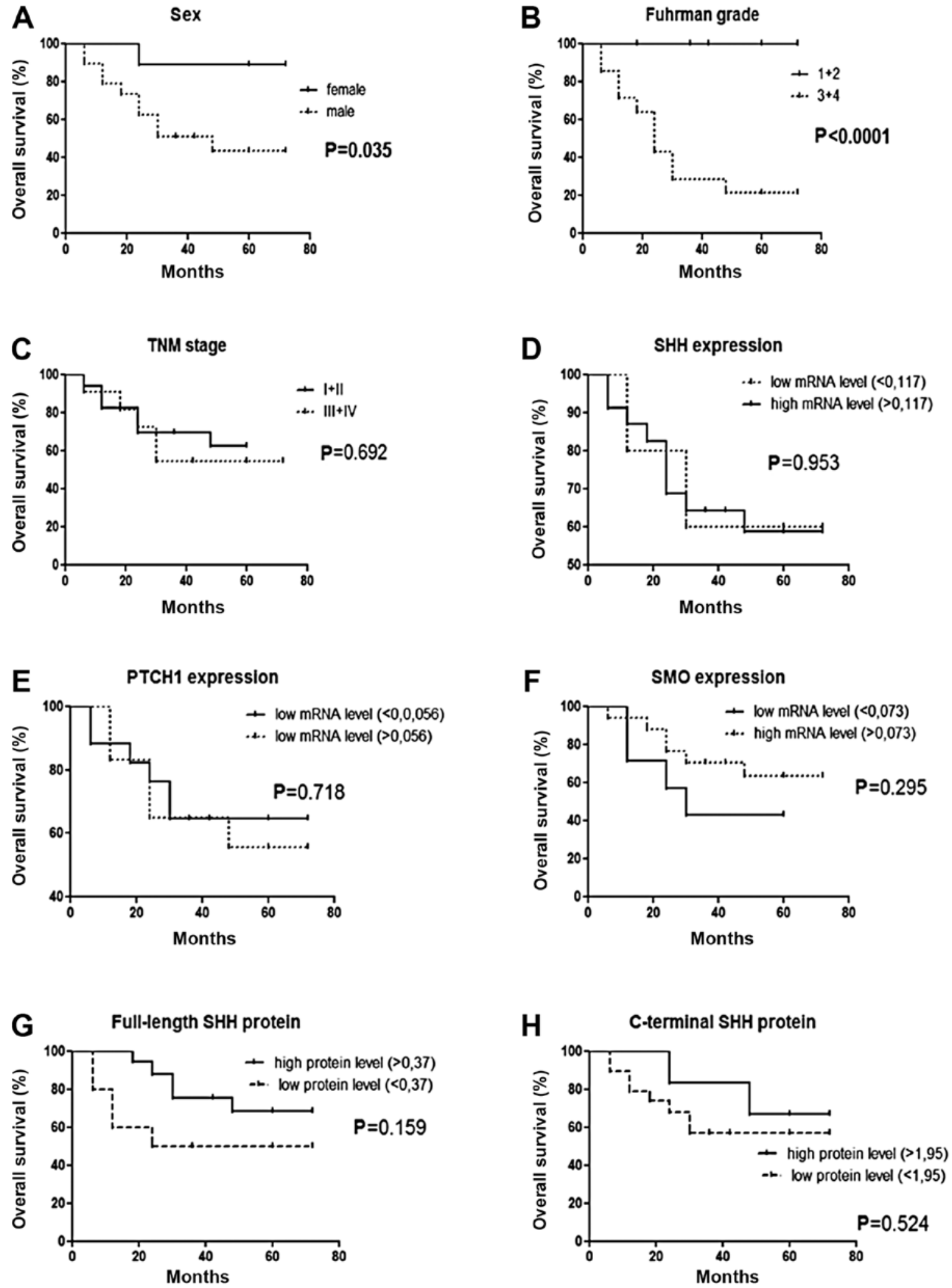

Figure 6. Kaplan-Meier's survival analysis for 28 patients with ccRCC related to (A, B, C) clinicopathological and (D, E, F, G, H) molecular data. The threshold value for each analyzed gene based on the median of (D) SHH, (E) PTCH1 and (F) SMO mRNA levels or (G) full-length and (H) C-terminal SHH protein levels in control samples. ccRCC, clear cell renal cell carcinoma; SHH, Sonic Hedgehog Signaling Molecule; PTCH1, Patched 1 Receptor; SMO, Smoothened, Frizzled Class G protein-coupled Receptor; GLI1, glioma-associated oncogene 1.

acts as an SMO protein inhibitor, decreases ccRCC cells proliferation and stimulates their apoptosis in vitro as well as in vivo (15). These findings suggest that, as in the BCC tumors, SMO may act as a potential drug target for ccRCC.
Among other types of cancers, increased content of SMO protein, assessed by the IHC method, was demonstrated by Ding et al in colon cancer tissues, obtained from 96 patients. Moreover, the level of SMO protein was positively related to 
the presence of lymph node metastases and higher $\mathrm{T}$ stages, which suggested the contribution of this gene in the colon cancer progression (30).

Glioma-Associated Oncogene 1 (GLI1), together with GLI2 and GLI3, are the members of zinc finger transcription factors family (10). Our study indicated a considerable increase of GLII mRNA level in cancer tissues, what is consistent with previous reports regarding ccRCC tissues $(15,16)$. Moreover, Furukawa et al, which assessed immunoreactivity of GLI1 and GLI2 proteins in ccRCC tissues derived from 39 patients, observed that strong GLI2 expression, but not GLI1, was correlated with a shorter period of progression free survival (31). Our results also did not indicate an association between GLII mRNA level and patients' survival (data not shown). Elevated GLI1 protein immunoreactivity, assessed by IHC staining of 204 tissue samples, was also observed in breast cancer cells and additionally it was correlated with unfavorable overall survival as well as higher tumor stage (32). Furthermore, increased GLI1 protein immunoreactivity was observed in the other tumor types such as the bladder (33) or ovarian cancers (34).

Our statistical analysis revealed that the expression rates of almost all the SHH pathway components in tumor tissues at the mRNA levels were correlated with each other. These findings suggest that $\mathrm{SHH}$ signaling is reactivated in ccRCC through canonical way, dependent on the amount of its upstream regulator, SHH protein (35) The same way of SHH pathway activation has been observed also in breast cancer (36) and non-small cell lung carcinoma cell lines (37), however, such suggestion needs confirmation based on in vitro studies with the use of RCC cell lines.

The association between the expression of SHH pathway components and cancer prognostic factors was reported in pancreatic adenocarcinoma (38), glioma (39) and other cancer types (24-26,30,32). Moreover, the expression profiles of SHH signaling genes in some cancer types correlated with the patients' overall survival $(14,40)$. Our statistical analysis did not reveal any relationships between $\mathrm{SHH}$ pathway genes mRNA level and ccRCC prognostic factors, however, most of the cited studies based on different techniques (IHC) and semi-quantification of SHH signaling proteins. Due to technical limitations, the IHC method could not be applied to our study, since the selection and antibodies and prior to IHC technique, western blot optimization took too long. Therefore, we plan to perform immunohistochemical studies in a larger cohort of ccRCC patients. There was also no correlation between the level of $S H H, P T C H 1$ and SMO mRNA as well as SHH proteins and patients' overall survival. Thus, according to our preliminary findings, observed changes in SHH pathway genes expression in tumor tissues probably are not associated with the ccRCC progression and patients' outcome.

In summary, increased expression of $\mathrm{SHH}$, SMO, Glil genes and full-length $\mathrm{SHH}$ protein level, as well as decrease of C-terminal SHH protein level in tumor ccRCC tissues, suggest the involvement of SHH signaling in ccRCC initiation.

\section{Acknowledgements}

Not applicable.

\section{Funding}

The current study was founded by ST-12 and ST-02-0117/07 statutory funds of the Medical University of Gdańsk.

\section{Availability of data and materials}

The datasets used and/or analyzed during the current study are available from the corresponding author on reasonable request.

\section{Authors' contributions}

AKC performed molecular analysis, performed the statistical tests and prepared the manuscript. JK acquired tissue samples and patient data and revised the manuscript. MM acquired tissue samples and patients' data and revised the manuscript. ZK substantially contributed to the interpretation of the results and revised the manuscript. PMW designed and supervised the study and revised the manuscript. All authors read and approved the final manuscript.

\section{Ethics approval and consent to participate}

The study received the approval of the Independent Bioethics Commission at the Medical University of Gdańsk (decision no. NKEBN/4/2011 and NKBBN/370/2016) and written consent was obtained before the surgery from each patient. All experimental procedures were performed according to the regulations and internal biosafety and bioethics guidelines.

\section{Patient consent for publication}

Not applicable.

\section{Competing interests}

The authors declare that they have no competing interests.

\section{References}

1. Hsieh JJ, Purdue MP, Signoretti S, Swanton C, Albiges L, Schmidinger M, Heng DY, Larkin J and Ficarra V: Renal cell carcinoma. Nat Rev Dis Primers 3: 17009, 2017.

2. Lu J, Zhu L, Zheng LP, Cui Q, Zhu HH, Zhao H, Shen ZJ, Dong HY, Chen SS, Wu WZ and Tan JM: Overexpression of ULK1 represents a potential diagnostic marker for clear cell renal carcinoma and the antitumor effects of SBI-0206965. EBioMedicine 34: 85-93, 2018.

3. Sanchez DJ and Simon MC: Genetic and metabolic hallmarks of clear cell renal cell carcinoma. Biochim Biophys Acta Rev Cancer 1870: 23-31, 2018.

4. Escudier B, Porta C, Schmidinger M, Rioux-Leclercq N, Bex A, Khoo V, Grünwald V, Gillessen S and Horwich A; ESMO Guidelines Committee: Renal cell carcinoma: ESMO clinical practice guidelines for diagnosis, treatment and follow-up $†$. Ann Oncol 30: 706-720, 2019.

5. Gupta S, Takebe N and LoRusso P: Targeting the hedgehog pathway in cancer. Ther Adv Med Oncol 2: 237-250, 2010.

6. Fattahi S,Pilehchian Langroudi M and Akhavan-Niaki H: Hedgehog signaling pathway: Epigenetic regulation and role in disease and cancer development. J Cell Physiol 233: 5726-5735, 2018.

7. Choudhry Z, Rikani AA, Choudhry AM, Tariq S, Zakaria F, Asghar MW, Sarfraz MK, Haider K, Shafiq AA and Mobassarah NJ: Sonic hedgehog signalling pathway: A complex network. Ann Neurosci 21: 28-31, 2014. 
8. Giroux Leprieur E, Tolani B, Li H, Leguay F, Hoang NT, Acevedo LA, Jin JQ, Tseng HH, Yue D, Kim IJ, et al: Membrane-bound full-length sonic hedgehog identifies cancer stem cells in human non-small cell lung cancer. Oncotarget 8: 103744-103757, 2017.

9. Skoda AM, Simovic D, Karin V, Kardum V, Vranic S and Serman L: The role of the hedgehog signaling pathway in cancer: A comprehensive review. Bosn J Basic Med Sci 18: 8-20, 2017.

10. Didiasova M, Schaefer L and Wygrecka M: Targeting GLI transcription factors in cancer. Molecules 23: E1003, 2018.

11. Buetti-Dinh A, Jensen R and Friedman R: A computational study of hedgehog signalling involved in basal cell carcinoma reveals the potential and limitation of combination therapy. BMC Cancer 18: 569, 2018

12. Kubo M, Nakamura M, Tasaki A, Yamanaka N, Nakashima H, Nomura M, Kuroki S and Katano M: Hedgehog signaling pathway is a new therapeutic target for patients with breast cancer. Cancer Res 64: 6071-6074, 2004.

13. Xu M, Li X, Liu T, Leng A and Zhang G: Prognostic value of hedgehog signaling pathway in patients with colon cancer. Med Oncol 29: 1010-1016, 2012.

14. Saze Z, Terashima M, Kogure M, Ohsuka F, Suzuki H and Gotoh M: Activation of the sonic hedgehog pathway and its prognostic impact in patients with gastric cancer. Dig Surg 29: $115-123,2012$

15. Dormoy V, Danilin S, Lindner V, Thomas L, Rothhut S, Coquard C, Helwig JJ, Jacqmin D, Lang H and Massfelder T: The sonic hedgehog signaling pathway is reactivated in human renal cell carcinoma and plays orchestral role in tumor growth. Mol Cancer 8: 123, 2009.

16. Zhou J, Zhu G, Huang J, Li L, Du Y, Gao Y, Wu D, Wang X, Hsieh JT, He D and Wu K: Non-canonical GLI1/2 activation by PI3K/AKT signaling in renal cell carcinoma: A novel potential therapeutic target. Cancer Lett 370: 313-323, 2016.

17. Behnsawy HM, Shigemura K, Meligy FY, Yamamichi F, Yamashita M, Haung WC, Li X, Miyake H, Tanaka K, Kawabata M, et al: Possible role of sonic hedgehog and epithelial-mesenchymal transition in renal cell cancer progression. Korean J Urol 54: 547-554, 2013.

18. Eble JN, Weltgesundheitsorganisation and International Agency for Research on Cancer: Pathology and genetics of tumours of the urinary system and male genital organs: Editorial and consensus conference in Lyon, France, December 14-18, 2002. IARC Press, Lyon, 2006.

19. Cancer Genome Atlas Research Network: Comprehensive molecular characterization of clear cell renal cell carcinoma. Nature 499: 43-49, 2013.

20. Wierzbicki PM, Klacz J, Rybarczyk A, Slebioda T, Stanislawowski M, Wronska A, Kowalczyk A, Matuszewski M and Kmiec Z: Identification of a suitable qPCR reference gene in metastatic clear cell renal cell carcinoma. Tumor Biol 35 12473-12487, 2014.

21. Livak KJ and Schmittgen TD: Analysis of relative gene expression data using real-time quantitative PCR and the 2(-Delta Delta C(T)) method. Methods 25: 402-408, 2001

22. Ma Y, Zhang P, Wang F, Yang J, Yang Z and Qin H: The relationship between early embryo development and tumourigenesis. J Cell Mol Med 14: 2697-2701, 2010.

23. Siegel R, Ward E, Brawley O and Jemal A: Cancer statistics, 2011: The impact of eliminating socioeconomic and racial disparities on premature cancer deaths. CA Cancer J Clin 61: 212-236, 2011

24. Jiang WG, Ye L, Ruge F, Sun PH, Sanders AJ, Ji K, Lane J, Zhang L, Satherley L, Weeks HP, et al: Expression of sonic hedgehog (SHH) in human lung cancer and the impact of YangZheng XiaoJi on SHH-mediated biological function of lung cancer cells and tumor growth. Anticancer Res 35: 1321-1331, 2015.
25. Bian X, Sun H, Xue H, Zhang G, Zhang CH, Liu XL, Su J and $\mathrm{Li} \mathrm{SJ}$ : Expression and clinical significance of Shh/Gli-1 in papillary thyroid carcinoma. Tumour Biol 35: 10523-10528, 2014.

26. Choe JY, Yun JY, Jeon YK, Kim SH, Choung HK, Oh S, Park M and Kim JE: Sonic hedgehog signalling proteins are frequently expressed in retinoblastoma and are associated with aggressive clinicopathological features. J Clin Pathol 68: 6-11, 2015.

27. Qi X, Schmiege P, Coutavas E, Wang J and Li X: Structures of human patched and its complex with native palmitoylated sonic hedgehog. Nature 560: 128-132, 2018

28. Lin T and Matsui W: Hedgehog pathway as a drug target: Smoothened inhibitors in development. Onco Targets Ther 5: 47-58, 2012

29. Xin M, Ji X, De La Cruz LK, Thareja S and Wang B: Strategies to target the Hedgehog signaling pathway for cancer therapy. Med Res Rev 38: 870-913, 2018.

30. Ding YL, Wang QS, Zhao WM and Xiang L: Expression of smoothened protein in colon cancer and its prognostic value for postoperative liver metastasis. Asian Pac J Cancer Prev 13 4001-4005, 2012

31. Furukawa J, Miyake $\mathrm{H}$ and Fujisawa M: GLI2 expression levels in radical nephrectomy specimens as a predictor of disease progression in patients with metastatic clear cell renal cell carcinoma following treatment with sunitinib. Mol Clin Oncol 5: 186-192, 2016.

32. ten Haaf A, Bektas N, von Serenyi S, Losen I, Arweiler EC, Hartmann A, Knüchel R and Dahl E: Expression of the glioma-associated oncogene homolog (GLI) 1 in human breast cancer is associated with unfavourable overall survival. BMC Cancer 9: 298, 2009.

33. He HC, Chen JH, Chen XB, Qin GQ, Cai C, Liang YX, Han ZD, Dai QS, Chen YR, Zeng GH, et al: Expression of hedgehog pathway components is associated with bladder cancer progression and clinical outcome. Pathol Oncol Res 18: 349-355, 2012.

34. Ciucci A, De Stefano I, Vellone VG, Lisi L, Bottoni C, Scambia G, Zannoni GF and Gallo D: Expression of the glioma-associated oncogene homolog 1 (Gli1) in advanced serous ovarian cancer is associated with unfavorable overall survival. PLoS One 8: e60145, 2013.

35. Carballo GB, Honorato JR, de Lopes GPF and Spohr TCLSE: A highlight on Sonic hedgehog pathway. Cell Commun Signal 16: $11,2018$.

36. Mukherjee S, Frolova N, Sadlonova A, Novak Z, Steg A, Page GP, Welch DR, Lobo-Ruppert SM, Ruppert JM, Johnson MR and Frost AR: Hedgehog signaling and response to cyclopamine differ in epithelial and stromal cells in benign breast and breast cancer. Cancer Biol Ther 5: 674-683, 2006.

37. Singh S, Wang Z, Liang Fei D, Black KE, Goetz JA, Tokhunts R, Giambelli C, Rodriguez-Blanco J, Long J, Lee E, et al: Hedgehog-producing cancer cells respond to and require autocrine hedgehog activity. Cancer Res 71: 4454-4463, 2011.

38. Maréchal R, Bachet JB, Calomme A, Demetter P, Delpero JR, Svrcek M, Cros J, Bardier-Dupas A, Puleo F, Monges G, et al: Sonic hedgehog and Glil expression predict outcome in resected pancreatic adenocarcinoma. Clin Cancer Res 21: 1215-1224, 2015.

39. Li Q, Zhang Y, Zhan H, Yuan Z, Lu P, Zhan L and Xu W: The Hedgehog signalling pathway and its prognostic impact in human gliomas. ANZ J Surg 81: 440-445, 2011.

40. Cheng J, Gao J and Tao K: Prognostic role of Gli1 expression in solid malignancies: A meta-analysis. Sci Rep 6: 22184, 2016.

This work is licensed under a Creative Commons Attribution-NonCommercial-NoDerivatives 4.0 International (CC BY-NC-ND 4.0) License. 